\title{
Simulation of magneto-rheological semi-active suspension system of EV driven by in-wheel motor
}

\author{
Shuanglong Zhu ${ }^{1,}$, ,Hailu Tang ${ }^{2, b}$ \\ School of Automotive Engineering,Wuhan University of Technology,Wuhan Hubei 430070, China \\ asolezsl@163.com, ${ }^{b} 742180200 . q q . c o m$
}

Keywords:EV driven by in-wheel motor;1/4 vehicle model;Magneto-rheological semi-active suspension system; Multi degree of freedom.

\begin{abstract}
Semi-active control of vehicle suspension system can be realized by adopting the magneto-rheological damper. This paper does research on EV driven by in-wheel motor,which adopts the motor suspension device and the magneto-rheological semi-active suspension. Based on the vehicle dynamics theory, the three degrees of freedom model of $1 / 4$ vehicle with magneto-rheological semi-active suspension and passive suspension are established respectively. Then the MATLAB/Simulink is used to simulate the two kinds of suspension systems. The magneto-rheological semi-active suspension adopts the "Sky-hook" control strategy. The simulation results show the advantages of magneto-rheological semi-active suspension. This paper provides a reference method for simulation with multi degree of freedom vibration system meanwhile.
\end{abstract}

\section{Introduction}

EV driven by in-wheel motor is a new electric vehicle, and the in-wheel motor takes place of the traditional engine and transmission system. Compared with ICE, it has many advantages [1] and has become the research focus of a new generation of electric vehicle technology. Purdy [2] points out that the unsprung mass of the vehicle increases due to the introduction of in-wheel motor, which enlarges the dynamic load of the tire and the vehicle body vibration acceleration, thus the tire grounding performance and car ride comfort get worse; Liang [3] designs a suspension device for in-wheel motor based on the dynamic vibration absorbing theory.

The suspension stiffness and the damping value of the active suspension system can be adaptively adjusted according to the change of the automobile driving conditions, so the suspension is always in the best damping state [4]. The semi-active suspension is one type of active suspension, which consist of variable parameters of the suspension spring and damper. It adjusts the suspension stiffness value or damping coefficient via a small amount of energy.

This paper does research on EV driven by in-wheel motor, which adopts the motor suspension device and the magneto-rheological semi-active suspension.Based on the vehicle dynamics theory, the three degrees of freedom model of $1 / 4$ vehicle with magneto-rheological semi-active suspension and passive suspension are established respectively.The MATLAB/Simulink is used to simulate the model.Finally the results of the simulation are analyzed in detail.

\section{Road input}

The road space roughness $\mathrm{Y}$ is the basic input for Car Ride Comfort and is the main reason causing the vehicle to generate vibrations. Random road can reflect the actual road condition when driving. The filtered white noise is suitable as the road input model. That is:

$$
\xi(t)=-2 \pi f_{0} y(t)+2 \pi \sqrt{G_{0} V} w(t)
$$


Where $\mathrm{y}(\mathrm{t})$ is road displacement; G0 is road roughness coefficient; $\mathrm{w}(\mathrm{t})$ is White Gauss noise, mean is zero; $\mathrm{V}$ is vehicle speed; and $\mathrm{f}_{0}$ is Cut-off frequency, $0.1 \mathrm{~Hz}$. When the vehicle drives on the level $\mathrm{C}$ road at the speed of $20 \mathrm{~m} / \mathrm{s}, \mathrm{G}_{0}=256 \times 10^{-6} \mathrm{~m}^{2} / \mathrm{m}^{-1}$.

\section{1/4 vehicle model with magneto-rheological semi-active suspension and passive suspension}

The $1 / 4$ vehicle model with magneto-rheological semi-active suspension/passive suspension is shown in figure 1(a) and figure 1(b).

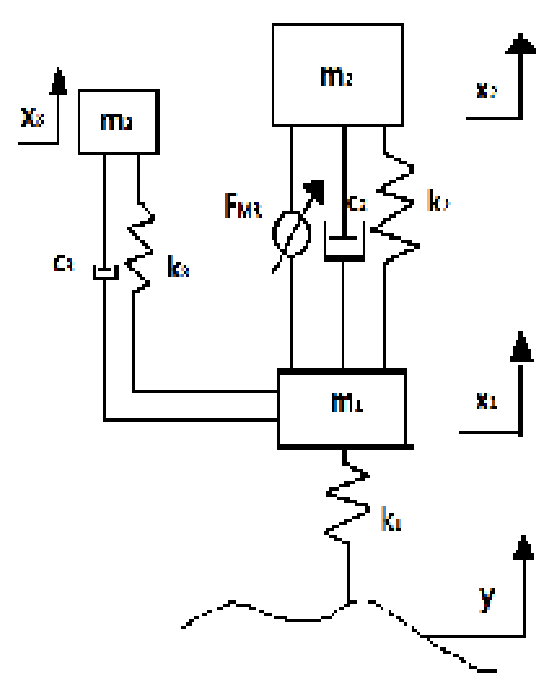

Figure 1(a). The 1/4 vehicle model with magneto-rheological semi-active suspension

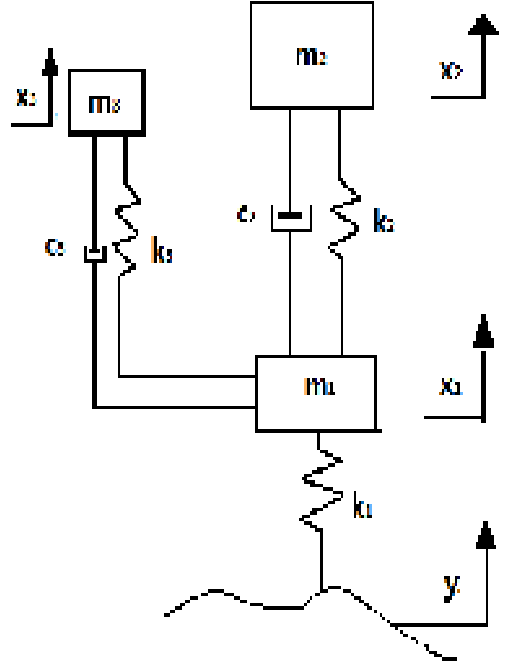

Figure 1(b). The 1/4 vehicle model with passive suspension model

Where:

$\mathrm{m}_{1}$ :Unsprung mass $\mathrm{m}_{2}$ :Sprung mass $\mathrm{m}_{3}$ :Motor mass $\mathrm{k}_{1}$ :Tire stiffness $\mathrm{k}_{2}$ :Suspension stiffness $\mathrm{k}_{3}$ :Motor suspension device stiffness $\mathrm{c}_{2}$ :Magneto-rheological damper inherent damping $\mathrm{c}_{3}$ :Motor suspension device damping $A_{\mathrm{P}}:$ Piston effective area D:Cylinder diameter L:Piston length h:Interval between Piston and Cylinder $\tau_{\gamma}$ :Shear yield stress $\tau_{\gamma}$ :Magneto-rheological fluid viscosity

$$
F_{M R}=\left(\frac{3 L A_{p}}{h}+\pi L D\right) \tau_{\gamma} \operatorname{sgn}[u(t)]=\left(\frac{3 L A_{p}}{h}+\pi L D\right) \tau_{\gamma} \operatorname{sgn}[\&-\&]
$$

For $1 / 4$ vehicle model with magneto-rheological semi-active suspension, the dynamics equation is established based on Newton's law:

$$
\begin{aligned}
& m_{1} \hat{\delta}+\left(c_{2}+c_{3}\right) \hat{x}_{1}-c_{2} \hat{x}_{2}-c_{3} \hat{x}_{3}+\left(k_{1}+k_{2}+k_{3}\right) x_{1}-k_{2} x_{2}-k_{3} x_{3}-F_{M R}=k_{1} y \\
& m_{2} \hat{\varepsilon}+c_{2}\left(\hat{x}_{2}-\hat{x}_{1}\right)+k_{2}\left(x_{2}-x_{1}\right)+F_{M R}=0 \\
& m_{3} \hat{x}_{3}+c_{3}\left(\hat{x}_{3}-\hat{x}_{1}\right)+k_{3}\left(x_{3}-x_{1}\right)=0
\end{aligned}
$$

State variables $\quad X=\left[\hat{x}_{1}, \hat{x}_{2}, \boldsymbol{x}_{3}, x_{1}, x_{2}, x_{3}\right]^{T}$

Input variables $U=\left[F_{M R}, y\right]^{T}$

Output variables $Y=\left[\mathfrak{s}_{2}, \boldsymbol{x}_{1}, x_{2}-x_{1}, x_{1}-y\right]^{T}$

The output variables are the vehicle body vertical acceleration, Unspring mass acceleration, Suspension dynamic deflection and Tire run-out. They are the evaluation index of the vehicle suspension system [5]. 
Dynamics equation is ransformed to state space equation:

$$
\left\{\begin{array}{l}
X^{£}=A X+B U \\
Y=C X+D U
\end{array}\right.
$$

Get coefficient matrix:

$$
\begin{gathered}
A=\left[\begin{array}{cccccc}
-\frac{c_{2}+c_{3}}{m_{1}} & \frac{c_{2}}{m_{1}} & \frac{c_{3}}{m_{1}} & -\frac{k_{1}+k_{2}+k_{3}}{m_{1}} & \frac{k_{2}}{m_{1}} & \frac{k_{3}}{m_{1}} \\
\frac{c_{2}}{m_{2}} & -\frac{c_{2}}{m_{2}} & 0 & \frac{k_{2}}{m_{2}} & -\frac{k_{2}}{m_{2}} & 0 \\
\frac{c_{3}}{1} & 0 & -\frac{c_{3}}{m_{3}} & \frac{k_{3}}{m_{3}} & 0 & -\frac{k_{3}}{m_{3}} \\
0 & 0 & 0 & 0 & 0 & 0 \\
0 & 1 & 0 & 0 & 0 & 0 \\
-\frac{1}{m_{2}} & 0 \\
0 & 0 \\
0 & 0 \\
0 & 0 \\
0 & 0
\end{array}\right] \\
B=\left[\begin{array}{cc}
m_{1} \\
\frac{k_{1}}{m_{2}}
\end{array}\right]
\end{gathered}
$$

For the 1/4 vehicle model with passive suspension, we can also establish the dynamics equation, transform dynamics equation to state space equation and get the coefficient matrix A.B.C.D in the same way.

\section{Sky-hook damping control strategy}

One common simi-active suspension control method is Sky-hook damping control strategy. The model is shown in figure 2. The damper is arranged between the sprung mass and the virtual inertia space [6]. The corresponding Sky-hook damping force is:

$$
F_{d}=C_{S k j} \hat{x}_{2}
$$

where, $\mathrm{C}_{\text {Sky }}$ is the Sky-hook damping coefficient and needs to be optimized according to the suspension system. 


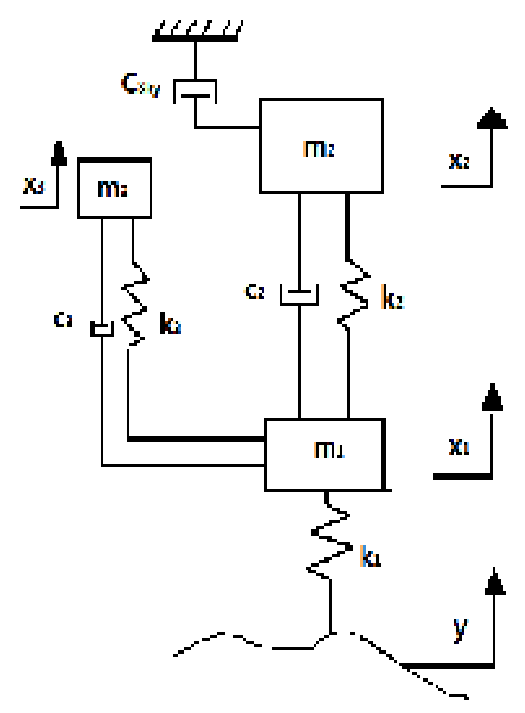

Figure 2. Sky-hook damping control strategy model

The dynamics equation is established in order to determine $\mathrm{C}_{\text {sky }}$ :

$$
\begin{aligned}
& m_{1} \approx+\left(c_{2}+c_{3}\right) \hat{x}_{1}-c_{2} \hat{k}_{2}-c_{3} \hat{x}_{3}+\left(k_{1}+k_{2}+k_{3}\right) x_{1}-k_{2} x_{2}-k_{3} x_{3}=k_{1} y \\
& m_{2} \mathfrak{k}_{2}+c_{2}\left(\hat{x}_{2}-\hat{x}_{1}\right)+k_{2}\left(x_{2}-x_{1}\right)+C_{S k y_{2}} \hat{k}_{2}=0 \\
& m_{3} \mathfrak{x}_{3}+c_{3}\left(\mathfrak{x}_{3}-\mathfrak{x}_{1}\right)+k_{3}\left(x_{3}-x_{1}\right)=0
\end{aligned}
$$

Laplace transform:

$$
\begin{aligned}
& {\left[m_{1} S^{2}+\left(c_{2}+c_{3}\right) S+\left(k_{1}+k_{2}+k_{3}\right)\right] X_{1}(S)-\left(c_{2} S+k_{2}\right) X_{2}(S)-\left(c_{3}+k_{3}\right) X_{3}(S)=k_{1} Y(S)} \\
& {\left[m_{2} S^{2}+\left(c_{2}+C_{S k y}\right) S+k_{2}\right] X_{2}(S)-\left[c_{2} S+k_{2}\right] X_{1}(S)=0} \\
& \left(m_{3} S^{2}+c_{3} S+k_{3}\right) X_{3}(S)-\left(c_{3} S+k_{3}\right) X_{1}(S)=0
\end{aligned}
$$

Transfer function of Tire:

$$
H_{1}=\frac{X_{1}(S)}{Y(S)}=\frac{k_{1}\left[m_{2} S^{2}+\left(c_{2}+C_{S k y}\right) S+k_{2}\right]}{\Delta S}
$$

Transfer function of car ride comfort :

$$
H_{2}=\frac{X_{2}(S)}{Y(S)}=\frac{k_{1}\left(c_{2} S+k_{2}\right)}{\Delta S}
$$

To maintain the ideal maneuverability, the wheel needs to follow the road surface profile under various conditions. The ideal suspension ride comfort requires the sprung mass remains stationary, so the ideal amplitude frequency characteristics of $\mathrm{H}_{1}$ and $\mathrm{H}_{2}$ should be almost horizontal and close to zero.

The curves of amplitude frequency characteristics of $\mathrm{H}_{1}$ and $\mathrm{H}_{2}$ are shown in Figure 3. The curves shows that the $\mathrm{H}_{2}$ is closer to the ideal amplitude frequency characteristics when $\mathrm{C}_{\text {Sky }}$ increases, while the tire run-out sharpens at the same time, which makes the control stability bad.Compromising on the suspension ride comfort and control stability, the reasonable $\mathrm{C}_{\text {Sky }}$ is $2000 \mathrm{Ns} / \mathrm{m}$.

To be equivalent to the Sky-hook damp, there is:

$$
F_{M R}=F_{d}
$$




$$
F_{M R}=\left(\frac{3 L A_{p}}{h}+\pi L D\right) \tau_{\gamma} \operatorname{sgn}[u(t)]=\left(\frac{3 L A_{p}}{h}+\pi L D\right) \tau_{\gamma} \operatorname{sgn}[\&<\alpha]
$$

When the signatures of $\hat{x}_{2}$ and $\left(\hat{x}_{2}-\hat{x}_{1}\right)$ are the same, $\mathrm{F}_{\mathrm{MR}}$ is equal to $\mathrm{F}_{\mathrm{d}}$.

Otherwise, in order to narrow the differences, $F_{\mathrm{MR}}$ should be zero. That is:

$$
F_{M R}=\left\{\begin{array}{cc}
F_{d}=C_{s} \hat{x}_{2} & \hat{x}_{2}\left(\hat{x}_{2}-\hat{x}_{1}\right) \geq 0 \\
0 & \hat{\boldsymbol{k}}_{2}\left(\hat{x}_{2}-\hat{x}_{1}\right)<0
\end{array}\right.
$$

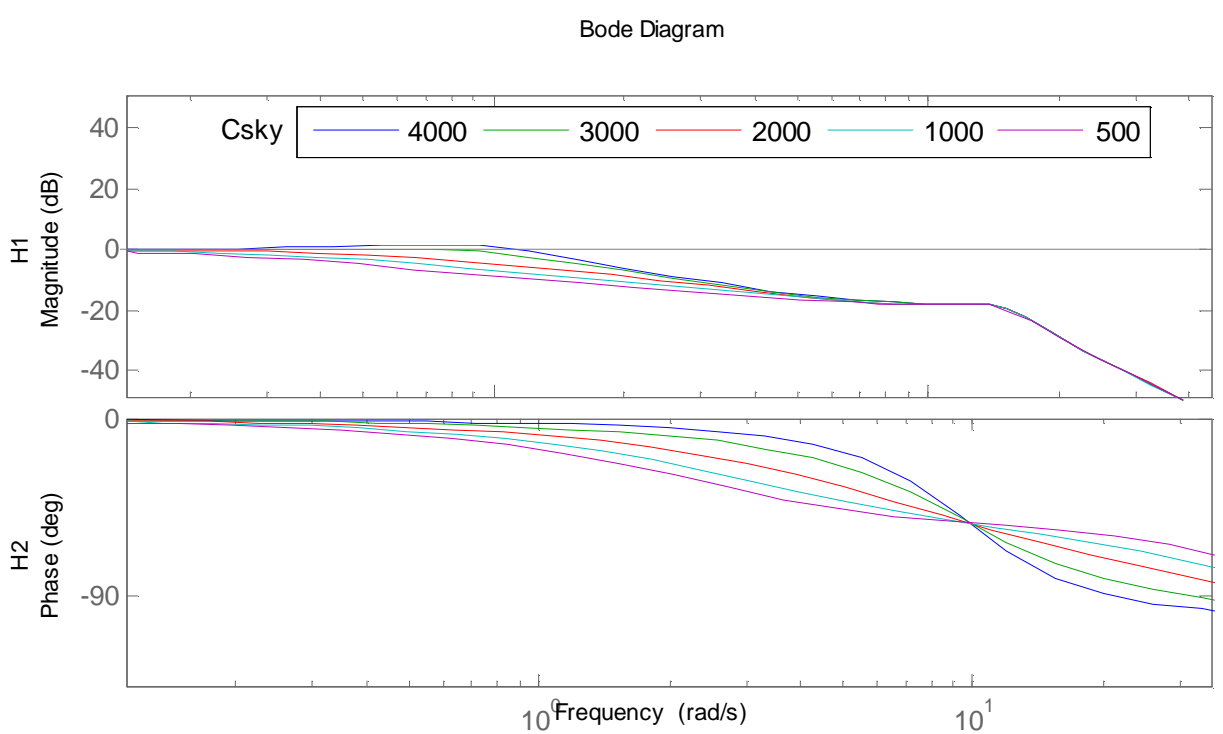

Figure 3. Curves of amplitude frequency characteristics of $\mathrm{H}_{1}$ and $\mathrm{H}_{2}$

\section{Simulation model}

Based on these two suspension models, Matlab/Simulink simulation model is shown figure 4 . The actual parameters of the model are as follows:

$m_{1}=17 \mathrm{~kg}, m_{2}=150 \mathrm{~kg}, m_{3}=28 \mathrm{~kg}, k_{1}=360000 \mathrm{~N} / \mathrm{m}, k_{2}=14740 \mathrm{~N} / \mathrm{m}, k_{3}=41000 \mathrm{~N} / \mathrm{m}, c_{2}=1190 \mathrm{~N} /(\mathrm{m} / \mathrm{s}), \mathrm{c}$ ${ }_{3}=600 \mathrm{~N} /(\mathrm{m} / \mathrm{s}), \mathrm{A}_{\mathrm{p}}=15 \mathrm{~cm}^{2}, \mathrm{D}=0.1 \mathrm{~m}, \mathrm{~L}=8.4 \mathrm{~cm}, \mathrm{~h}=2 \mathrm{~mm}, \tau_{\gamma}=60 \mathrm{kPa}, \eta=1.3 \mathrm{~Pa} \cdot \mathrm{s}$.

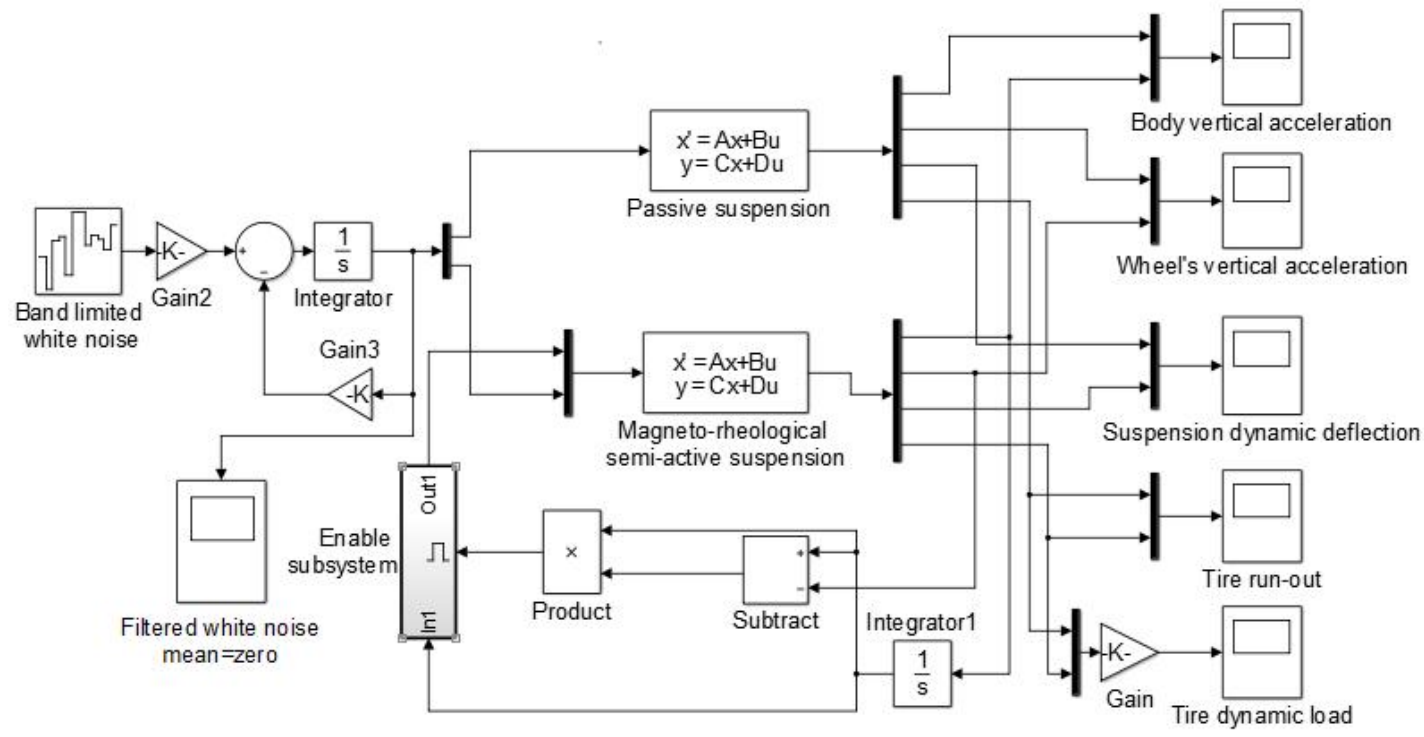

Figure 4. Matlab/Simulink simulation model of two suspension systems 


\section{Results and discussion}

The response curves of vehicle body vertical acceleration, suspension dynamic deflection, the tire dynamic load and the tire runout are shown in Figure 5 8.

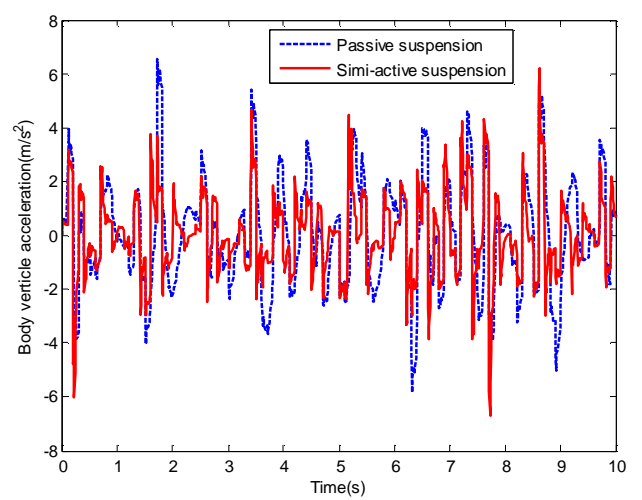

Figure 5. Vehicle body vertical acceleration

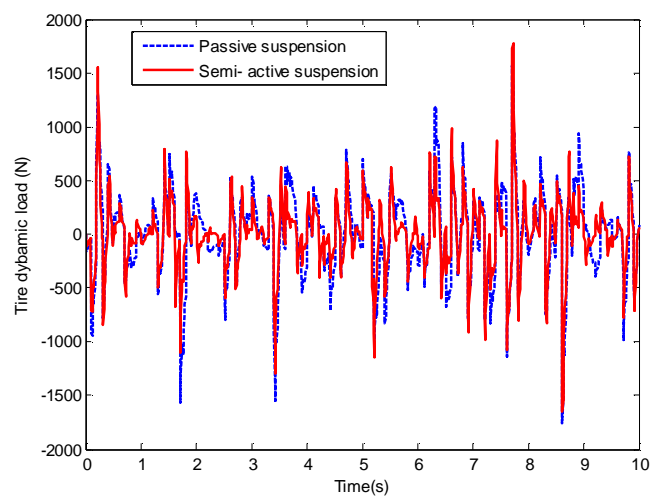

Figure 7. Tire dynamic load

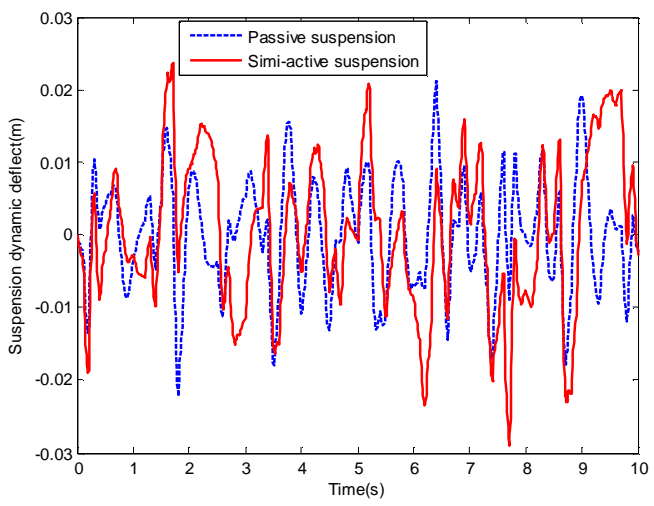

Figure 6. Suspension dynamic deflection

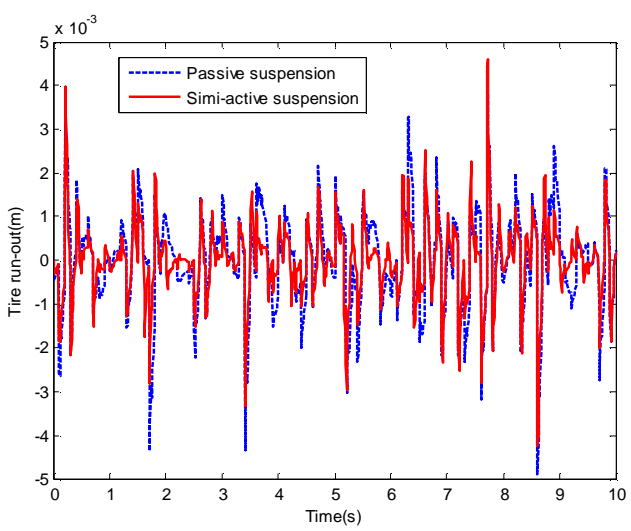

Figure 8. Tire run-out

Compared with passive suspension,the simi-active suspension improves the vehicle body vertical acceleration apparently,restrains the tire run-out,and enhances the adhesion performance of the tire.

\section{Conclusions}

Compared with the passive suspension system, the magneto-rheological semi-active suspension system with the "Sky-hook" control strategy improves the car ride performance and comfort obviously. We take the vertical three degree of freedom system of EV driven by in-wheel motor based on the dynamic vibration absorbing theory as an example, establish the dynamics equation and transform dynamics equation to state space equation further more, finally implement the simulation. This process provides a reference method for similar vibration system simulation with multi degree of freedom.

\section{Reference}

[1]Murata S.Vehicle Dynamics Innovation with In-Wheel Motor.SAE Paper 2011-39-7204.

[2]Purdy J. D.A brief investigation into the effect on suspension motions of high unspung mass.Journal of battlefield technology.2004,7(1):15-20.

[3] R.Liang,Z.P.Yu ,G.B.Ning.The suppression of negative effect of in-wheel drive

EV vertical vibration based on vibration absorption principle.JOURNAL OF MACHINE DESIGN.2008,1(25):28-30.

[4]G.L.Wang,L.Gu,F.C.Sun.The situation and trend of the technology of vehicle active 
suspension.ACTA ARMAMENTARII.2008,(8):80-82.

[5]Z.S.Yu.Automobile theory.Beijing:CHINA MACHINE PRESS,2009.3.

[6]M.Yu.Research on the control system of automobile magneto-rheological semi-active suspension[D].University Of Chongqing,2005 